\title{
Philosophiques
}

\section{La vision comme procédé de communication dans le Discours de la Méthode}

\section{André Vidricaire}

Volume 15, numéro 1, printemps 1988

URI : https://id.erudit.org/iderudit/027037ar

DOI : https://doi.org/10.7202/027037ar

Aller au sommaire du numéro

Éditeur(s)

Société de philosophie du Québec

ISSN

0316-2923 (imprimé)

1492-1391 (numérique)

Découvrir la revue

Citer cet article

Vidricaire, A. (1988). La vision comme procédé de communication dans le Discours de la Méthode. Philosophiques, 15(1), 95-105.

https://doi.org/10.7202/027037ar

\section{Résumé de l'article}

Le Discours de la Méthode oppose explicitement « faire voir " à «enseigner». À ce propos il s'agit ici de déterminer la fonction et la signification de ces verbes dans le contexte de la communication d'un savoir. Ce Discours, plutôt que de simplement transmettre des principes généraux, veut, par le truchement du tableau d'une vie présenté sous la forme d'une " histoire " ou d'une " fable ", susciter chez le lecteur l'idée correspondante de la recherche et de la découverte par soi-même. Aussi, sur le plan discursif, la première catégorie verbale, au lieu d'être une attribution est une attribution différée. Ce procédé sémiotique correspond à la thèse cartésienne selon laquelle l'acquisition d'une vérité implique examen et approbation de la part du destinataire. 


\title{
LA VISION COMME PROCÉDÉ DE COMMUNICATION DANS LE DISCOURS DE LA MÉTHODE
}

\author{
par André Vidricaire
}

\begin{abstract}
RÉSUMÉ. Le Discours de la Méthode oppose explicitement «faire voir» à «enseigner». À ce propos il s'agit ici de déterminer la fonction et la signification de ces verbes dans le contexte de la communication d'un savoir. Ce Discours, plutôt que de simplement transmettre des principes généraux, veut, par le truchement du tableau d'une vie présenté sous la forme d'une «histoire» ou d'une "fable », susciter chez le lecteur l'idée correspondante de la recherche et de la découverte par soi-même. Aussi, sur le plan discursif, la première catégorie verbale, au lieu d'être une attribution est une attribution différée. Ce procédé sémiotique correspond à la thèse cartésienne selon laquelle l'acquisition d'une vérité implique examen et approbation de la part du destinataire.
\end{abstract}

ABSTRACT. The Discours de la Méthode explicitely opposes "to show" to "to teach". In this connection, we want here to determine the function and the meaning of these verbs in the context of the transmission of knowledge. The Discours, instead of simply conveying general principles, endeavors, through the picture of a life displayed as a "history" or "fable", to give rise to the corresponding ideas of research and discovery in the reader himself. On the discursive level, the first verbal category, far from naming the ascription of an achieved state, must be understood as the delayed attribution of that state. This semiotic procedure corresponds to the Cartesian thesis according to which the acquisition of a truth involves examination and approval on the part of the addressee.

À première vue', le Discours de la Méthode se présente comme une œuvre philosophique très facile à lire. Mais quand on

1. Ce texte est une version remaniée de l'exposé présenté à l'ACFAS en mai 1987. 
se livre à son analyse, on y découvre tellement d'embûches qu'E. Gilson a songé en 1924 à en faire un commentaire historique ${ }^{2}$. Sa recherche a consisté à déterminer la nature exacte des faits rapportés dans cette œuvre de même qu'à donner le sens précis des mots, des expressions et des thèses philosophiques qui ne sont très souvent qu'esquissées. Or, même si ce commentaire de Gilson est devenu une référence obligée pour qui veut interpréter la pensée de Descartes, le Discours de la Méthode continue de soulever des problèmes de lecture. Il en est un que j'aimerais ici exposer et discuter. Il s'agit du problème de la communication d'un savoir : au lieu d'enseigner la méthode, Descartes choisit un procédé de transmission beaucoup plus complexe qui consiste à «faire voir » les démarches qu'il a faites et ce qu'il a finalement trouvé. Nous verrons que ce procédé, loin d'être un artifice rhétorique externe à la philosophie cartésienne, trouve son prolongement dans le mot "méditation» et correspond au contenu discuté.

\section{POSITION DU PROBLÈME}

Nous le savons tous, c'est très consciemment et très délibérément que Descartes appelle cet ouvrage DISCOURS de la Méthode et non pas TRAITÉ. Comme il s'en explique à Mersenne ${ }^{3}$, Huygens ${ }^{4}$, et Vatier ${ }^{5}$, il aurait choisi le mot «traité » si son dessein avait été d'enseigner la méthode. Mais, comme il ne voulait pas enseigner cette méthode, mais seulement en parler, en dire quelque chose, il a préféré le mot « discours » qui équivaut à «préface » ou «avis» touchant cette méthode. Or, parler de la méthode va consister pour Descartes à «faire voir les chemins qu'il a suivis ", à «montrer » comment il a conduit sa raison, à « représenter sa vie comme en un tableau ». Ainsi, cet écrit se présente comme une « histoire », voire même comme une « fable ». Enfin, La Dioptrique, Les Météores et La Géométrie sont des essais de cette méthode

2. Étienne GLLSON : Discours de la Méthode de René Descartes, texte et commentaires, $3^{e}$ édition, Paris, Vrin, 1962. Voir aussi du même auteur : "Projet d'un commentaire historique du Discours de la Méthode”(et discussions), Bulletin de la Société Française de Philosopbie, déc. 1924, pp. 135-150.

3. À Mersenne, mars 1637.

4. À Huygens, 25 février 1637.

5. À Vatier, 22 février 1638 ; aussi à XXX, avril 1637. 
parce que, dit-il, «je prétends que les choses qu'ils contiennent n'ont pu être trouvées sans elle et qu'on peut connaître par eux ce qu'elle vaut ${ }^{6}$. De même, les sujets de métaphysique, de physique et de médecine qui se trouvent dans le Discours, montrent que la méthode «s'étend à toutes sortes de matières » ${ }^{7}$. En résumé, Descartes oppose explicitement :

\section{$\left.\begin{array}{l}\text { DISCOURS } \\ \text { AVIS } \\ \text { PRÉFACE }\end{array}\right\}$ vs TRAITÉ}

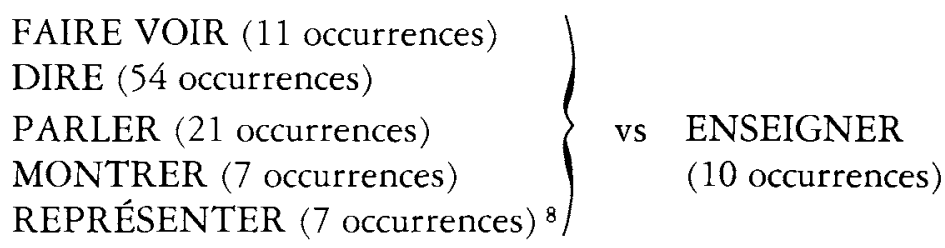

Certes, des circonstances historiques comme la condamnation de Galilée peuvent expliquer ces oppositions et le choix effectué par Descartes. Mais cette seule interprétation laisse croire que le Discours est une ouvre philosophique mineure en comparaison des Règles, du Monde et surtout des Méditations. En effet, selon ce point de vue, Descartes aurait livré un simple récit intellectuel accessible aux âmes bien nées en attendant des circonstances plus favorables pour communiquer son vrai système philosophique. Mais si nous étudions avec minutie la signification des mots « faire voir » et «représenter » et leur fonction dans l'économie générale du système philosophique de Descartes alors en formation, nous découvrirons que ce qui se présente comme une « histoire» ou une «fable» est une forme discursive en parfaite concordance avec les thèses philosophiques. Pour en établir la démonstration, il faut apporter des réponses à la fois d'ordre philosophique et d'ordre sémiotique aux questions suivantes : par exemple, comment expliquer que Descartes se refuse à enseigner la méthode qui lui a pourtant permis de trouver des démonstrations métaphysiques plus évidentes que les démonstrations de géométrie? À l'opposé,

6. À Mersenne, mars 1637 .

7. À XXX, avril 1637 .

8. Liste des occurences fournie par P.A. CaHNÉ: Index du Discours de la Métbode de René Descartes, Rome, 1977. 
que veut dire «faire voir» ou «montrer»? En quoi ce type de communication est moins sujet à induire en erreur qu' « enseigner » ? Comment Descartes peut-il proposer au lecteur une « histoire » ou une «fable» quand lui-même critique dans le cours d'études reçu au Collège de Laflèche l'histoire et les fables ? Comment justifier ce recours au récit historique quand la saisie intuitive du vrai est instantanée? Comment expliquer ce privilège accordé à l'œil quand les sens sont discrédités? Comment Descartes s'y prend-il pour rendre visible? Enfin, est-ce que ce procédé est spécifique au Discours ou se retrouve-t-il dans les Méditations?

\section{ENSEIGNER}

En tout premier lieu, signalons que ce Discours sur la Méthode ne pourra jamais devenir un «Traité de la Méthode», pour la simple raison que la méthode pour trouver le vrai ne peut pas s'enseigner. Comme l'écrit Gilson ${ }^{9}$, la méthode se ramène à quatre préceptes qui concernent deux opérations de l'esprit qui sont l'intuition et la déduction. Or, ces deux opérations ne consistent que dans l'usage spontané de notre lumière naturelle: elles ne peuvent donc pas être enseignées.

En second lieu, si Descartes se refuse à enseigner, c'est parce que l'enseignement considéré comme la transmission d'un savoir même vrai n'est pas une méthode d'acquisition de la vérité ${ }^{10}$. L'acquisition de la vérité implique un examen par l'intelligence et une approbation par la volonté. Même dans les cas où il est préférable de rechercher le conseil des plus habiles, Descartes veut qu'on « use de son propre jugement pour examiner leurs opinions ». Bref, même au niveau de l'agir, il se refuse à ce qu'on se laisse " conduire aveuglément par l'exemple» ${ }^{11}$. Voilà pourquoi le Discours qui est proposé néanmoins comme une «fable» laisse le lecteur libre de choisir les exemples à imiter ${ }^{12}$.

Sur le plan discursif, il faut noter que dans le Discours de la Méthode, Descartes ne se présente jamais en position d'émetteur

9. GILSON, id., p. $195-196$

10. GILSON, id., p. 167.

11. À Élizabeth, 18 août 1645.

12. $D M 0415$. 
d'un enseignement. Bien au contraire, au niveau de l'histoire ou de l'énoncé, il est récepteur d'un enseignement qui s'avère un échec:

— "Jai été nourri aux lettres dès mon enfance ${ }^{13}$.

— «Et même, ne m'étant pas contenté des sciences qu'on nous enseignait ${ }^{14}$.

- «Ils (les écrits des anciens païens) n'enseignent pas assez à les (les vertus) connaître ${ }^{15}$.

Nul doute que cette expérience qui lui a été personnellement désavantageuse, l'a conduit à chercher et à établir avec son lecteur une forme nouvelle de communication. Ce faisant, Descartes va transformer le schéma traditionnel de l'échange:

Position antérieure

enseigner $\rightarrow$ apprendre
Position nouvelle

faire voir $\rightarrow$ se persuader

\section{FAIRE VOIR, MONTRER}

Il faut signaler, au départ, que Descartes a étudié et analysé avec une très grande minutie les problèmes de la lumière, de la perception visuelle et des instruments comme le télescope pour améliorer la puissance de la vue. Il appelle son premier traité de physique Le Monde ou Traité de la Lumière. Or, tout en rédigeant ce traité sur la nature des choses matérielles, il écrit en même temps La Dioptrique qui vise à trouver un fondement scientifique aux applications pratiques des lunettes. Descartes justifie cet objectif par les propos suivants:

«Toute la conduite de notre vie dépend de nos sens, entre lesquels celui de la vue étant le plus universel et le plus noble, il n'y a point de doute que les inventions qui servent à augmenter sa puissance, ne soient des plus utiles qui puissent être ${ }^{16}$.

De même, dans son épistémologie et dans sa métaphysique, beaucoup de concepts philosophiques tirent leur origine d'une référence à la lumière dans son rapport à la vision. Ainsi, il parle de la «lumière naturelle» qui fonde l'évidence et la clarté des

\footnotetext{
13. DM 0421.

14. $D M 0505$

15. DM 0805 .

16. Diop. I.
} 
idées ${ }^{17}$. En outre, l'intuition est une saisie d'un seul regard, une perception dans l'instant d'une idée simple. Comme le montre Serres, ce modèle de l'intuition se réfère au modèle de la vision oculaire lequel s'appuie sur le modèle du tact ${ }^{18}$. Certes, l'action d'un objet extérieur excite l'organe de la vue auquel correspond instantanément sur le fond de l'œil une image rétinienne. Mais l'enjeu est de bien comprendre la définition que donne Descartes de l'image et de la perception. L'œil est comme des lentilles. Il possède trois humeurs par où passent par réfraction les rayons de la lumière pour pénétrer jusqu'au fond de l'oil où sont les petits filets du nerf optique. Dans les filets indépendants reliés au cerveau circulent les esprits animaux qui sont un air très subtil issu de la glande pinéale, siège de l'imagination et du sens commun. Ainsi, sous l'action de la lumière qui cause quelques mouvements sur les filets, les esprits animaux coulent par le nerf optique dans les muscles pour en modifier la figure et causer ainsi les mouvements de l'œil. En même temps, les diverses façons dont les filets sont pressés par les rayons tracent en la superficie intérieure du cerveau, comme dans la glande pinéale, une figure, une image ou une "peinture" semblable à l'objet extérieur qui a causé ces mouvements 19 .

Mais signalons que pour Descartes cette ressemblance est fort limitée et remplie de défauts ${ }^{20}$. La grandeur de l'image au fond de l'ail n'est pas la grandeur de l'objet et «ses parties sont renversées » ${ }^{21}$. L'image de sa figure est composée d'ovales et de losanges pour représenter les cercles et les carrés. Etc. D'autre part, ce n'est pas l'œil, mais plutôt l'âme qui voit la grandeur, la figure, la situation, la distance, la lumière et la couleur d'un objet. En effet, comme la matière et la pensée s'excluent l'une l'autre et

17. Med. 3

18. M. SERRES: «L'évidence, la vision et le tact», Études Philosophiques, 1968, n. 23, p. 191-195.

19. « Dans le cas de la perception visuelle, l'image rétinienne de l'objet, c-à-d. l'image qui y correspond dans le cerveau, et donc dans la glande pinéale, entretient une relation de similitude quantitative avec l'objet (voir A.T. IV, 130), l'idée ne représente pourtant pas la chose, d'après un principe de similitude qualitative ", W. RoD : «L'argument du rêve dans la théorie cartésienne de l'expérience», Les Études Philosophiques, n. 4, 1976, p. 464.

20. Diop. 5.

21. Id. 
comme l'image n'est finalement pour Descartes qu'une «chose corporelle... produite de l'action des corps extérieurs sur notre propre corps par l'intermédiaire des sens et des nerfs » ${ }^{22}$, il s'ensuit que seule la pensée perçoit. En d'autres termes, ce n'est pas par le moyen de l'image et de sa plus ou moins grande ressemblance avec l'objet qu'il y a perception, mais plutôt à l'occasion d'une excitation sur l'œil qui suscite quelque mouvement corporel qu'apparaissent dans l'âme des idées innées. "Les mouvements sont comme des signes qui provoquent dans l'âme certains sentiments » ${ }^{23}$ de lumière, de couleur, etc. En résumé, la perception pour Descartes est une connaissance d'un objet au moyen d'une idée qui le représente, tandis que l'image n'est, répétons-le, qu'une chose matérielle qui suscite en l'âme l'idée qui représente l'objet. Quelles sont les conséquences de cette théorie de l'image et de la perception sur le Discours de la Méthode? En surface, ce texte est proposé comme un «tableau» susceptible de susciter dans la pensée du lecteur une perception visuelle correspondante. Il s'agit du tableau d'une vie présentée sous la forme d'une «histoire» ou d'une "fable». Descartes précise que, se refusant à communiquer un ensemble de préceptes généraux, il décrit sous la forme d'un récit sa propre démarche de recherche et surtout les diverses découvertes que permet la méthode notamment dans les secteurs de la métaphysique, de la physique, de la médecine. En résumé, ce texte n'est qu'une (re) présentation d'images «concrètes", mais qui peuvent être l'occasion de susciter dans l'âme du lecteur l'idée correspondante de la recherche et de la découverte par soi-même. Ce texte rempli d'exemples est finalement une action qui occasionne, chez le lecteur, l'examen des idées qui les représente et peut-être leur approbation. La saisie de la vérité qui demeure à la charge du récepteur, est médiatisée, ici, par le récit d'un chemin qui y conduit et qui existe en tout homme.

Il faut noter que cette médiation n'est pas spécifique au Discours de la Méthode. Un choix similaire trouve son prolongement dans la forme rédactionnelle des Méditations. En effet, comme l'a établi J.P. Brodeur ${ }^{24}$, les Méditations avec ses procédés de « reprise»,

22. J.P. Sartre, L'imagination, Paris, PUF, 6e éd., 1965, p. 7.

23. Id., p. 8.

24. J.P. Brodeur, «Thèse et performance dans les "Méditations" de Descartes », Dialogue, 14,1975 , p. $51-79$. 
d'《 exercice » et de « modélisation » contiennent moins un ensemble de thèses philosophiques qu'une "opération scripturaire qui veut accomplir un geste de transformation sur le lecteur ${ }^{25}$. Ainsi comme le Discours, les Méditations sont une «description d'un processus de recherche et de découverte ${ }^{26}$.

Connaissant la fonction de "faire voir» dans l'économie générale du Discours, il nous faut maintenant déterminer sa signification. Pour ce faire, voici la liste des onze occurrences de «faire voir» 27 :

1. «Mon dessein n'est pas d'enseigner ici la méthode... mais seulement de faire voir en quelle sorte j'ai tâché de conduire la mienne». (0407-0409)

2. «Mais je serai bien aise de faire voir en ce discours, quels sont les chemins que j'ai suivis » (0331-0401)

3. «Que si mon ouvrage m'ayant assez plu, je vous en fais voir ici le modèle, ce n'est pas pour cela que je veuille conseiller à personne de l'imiter » (1507-1509)

4. «Et peut-être aussi en faisant voir les raisons que javais de douter de beaucoup de choses que les autres estiment certaines, plutôt qu'en me vantant d'aucune doctrine» (3025-3028)

5. "Je serais bien aise de poursuivre, et de faire voir ici toute la chaîne des autres vérités que j'ai déduites de ces premières " (4021-4023)

6-7 «De plus, je fis voir quelles étaient les lois de la nature » (4305-4306)... « je tâchai à démontrer toutes celles dont on eût pu avoir quelque doute, et à faire voir, qu'elles sont telles, qu'encore que Dieu avait créé plusieurs mondes, il n'y en saurait avoir aucun où elles manquassent d'être observées 》 (4308-4312)

8. "Mais parce que je n'en avais pas encore assez de connaissance pour en parler du même style que du reste, c.a.d. en démontrant les effets par les causes et faisant

25. Id., p. 51.

26. Id., p. 51.

27. P.A. Cahné: Index du Discours de la Méthode de René Descartes, Rome, 1977. 
voir de quelles semences et en quelle façon la nature les doit produire » (4525-4530)

9. «Et si je m'étais ici particulièrement arrêté à faire voir que, s'il y avait de telles machines, qui eussent les organes et la figure d'un singe 》 (5610-5612)

10. «J'avais décrit après cela l'âme raisonnable et fait voir qu'elle ne peut aucunement être tirée de la puissance de la matière » (5908-5910)

11. «Car elles m'ont fait voir qu'il est possible de parvenir à des connaissances qui soient fort utiles à la vie » (61286129).

Pour saisir le sens de «faire voir », il y a deux aspects qu'il importe de considérer. Premièrement, reprenant la distinction de Benveniste entre "histoire» et «discours》, "faire voir» est utilisé de manière équivalente dans les deux champs. En effet, dans les parties 1, 2, 5 « faire voir » est en contexte de discours et dans les parties 3 et 5 en contexte d'histoire. En conséquence «faire voir » ne peut pas, comme le prétend S. Romanowski, avoir le sens de « récit qui fait voir par opposition à une parole qui dit les idées directement ${ }^{28}$. Il y a des paroles qui font voir et ce, dès les parties 1 et 2 du Discours ${ }^{29}$. Il en va de même pour l'usage du verbe «montrer » situé, lui aussi, sur le plan de la spatialité.

À l'inverse, les verbes «parler» et «dire» qui sont des catégories de la temporalité n'appartiennent pas automatiquement à la catégorie du discours. Ainsi quand Descartes écrit à Mersenne que son dessein n'est pas d'enseigner la méthode, mais seulement d'en parler ${ }^{30}$, ce propos rejoint celui qui ouvre la quatrième partie du Discours :

"Je ne sais si je dois vous entretenir des premières méditations que j'y ai faites... toutefois, afin qu'on puisse juger si les fondements que j'ai pris sont assez fermes, je me trouve en quelque façon contraint d'en parler ${ }^{31}$.

28. S. Romanowski, L'Illusion chez Descartes, Paris, Klincksieck, 1974, p. 112.

29. Id., p. 128.

30. À Mersenne, 1630

31. DM 3114-3120. 
Or, cette «parole » prend la forme d'un récit et plus précisément celle d'une histoire de la découverte des principes métaphysiques.

Cela posé, l'enjeu consiste ici à déterminer la signification de ces verbes, quel que soit leur contexte. Comme le schéma suivant le résume, ils s'opposent tous aux verbes « enseigner », " conseiller », «donner des préceptes»:

$$
\text { ENSEIGNER vs }\left\{\begin{array}{l}
\text { FAIRE VOIR MONTRER } \\
\text { PARLER DIRE } \\
\text { aussi bien en } \\
\text { contexte de dis- } \\
\text { cours que d'his- } \\
\text { toire }
\end{array}\right.
$$

Au niveau discursif le verbe «enseigner» est l'attribution d'un objet d'un destinateur à un destinataire. Il n'en va pas de même pour le verbe «faire voir ». Comme l'écrit L. Panier, à partir de l'exemple de Jésus au désert où le diable MONTRE à Jésus "tous les royaumes du monde avec leur gloire»,

« cette monstration recouvre une attribution différée de l'objet sous la modalité cognitive du regard. De la vue à la possession, il y a toute la distance de la virtualité à la réalisation, tout l'espace d'un programme narratif à effectuer ${ }^{32}$.

En effet, le «faire voir» est une communication qui au lieu de simplement transmettre un objet (ou une performance) ne fait que le «montrer» et donc en diffère son attribution. Mais en même temps, cette forme de communication est un acte de persuasion qui vise à susciter chez le lecteur le désir d'avoir l'objet qui est montré. Panier présente comme suit le fonctionnement du «dire » et du «montrer» dans la persuasion:

32. L. PANIER : Récit et commentaires de la tentation de Jésus au désert, Paris 1984, p. 29. 
faire communicatif 33

\begin{tabular}{|c|c|c|}
\hline \multicolumn{2}{|c|}{$\begin{array}{l}\text { visuel } \\
\text { («montrer» - «faire voir») } \\
\text { spatialité - }\end{array}$} & $\begin{array}{r}\text { verbal } \\
\text { («parler»_ «dire ») } \\
\end{array}$ \\
\hline Thématisation & Attribution différée & Attribution réalisée \\
\hline $\begin{array}{l}\text { spatiale } \\
\text { (vision) }\end{array}$ & $\begin{array}{l}\text { faire voir } \\
\text { montrer } \\
\text { représenter }\end{array}$ & \\
\hline $\begin{array}{l}\text { temporelle } \\
\text { (audition) }\end{array}$ & $\begin{array}{l}\text { dire } \\
\text { parler } \\
\text { faire entendre }\end{array}$ & $\begin{array}{l}\text { enseigner } \\
\text { conseiller } \\
\text { donner des préceptes }\end{array}$ \\
\hline
\end{tabular}

\section{EN GUISE DE CONCLUSION}

En résumé, si Descartes se refuse à enseigner, c'est parce que la méthode elle-même ne s'enseigne pas et surtout parce qu'il n'y a pas d'appropriation sans examen ni approbation. Aussi choisit-il d'exciter la pensée «visuelle » du lecteur à l'aide de signes ou un tableau. Pour ce faire, il utilise un procédé discursif correspondant à cette démarche cognitive qui consiste à sélectionner des verbes comme «faire voir », « montrer», « représenter», «parler », dont la fonction est de différer la communication de règles ou de préceptes et de susciter chez le lecteur le désir de s'approprier après examen et jugement ce qui peut lui être utile. Ce faisant, Descartes évite que le lecteur de cette «histoire» ou de cette «fable» se laisse conduire aveuglément par les exemples qui lui sont présentés.

Ainsi, à la différence d' « enseigner » qui consiste à transmettre des thèses philosophiques avec leur démonstration (explications et preuves), «faire voir » consiste plutôt à décrire sous la forme d'une représentation notamment visuelle la démarche de recherche et de découverte correspondante.

Département de philosophie, Université du Québec à Montréal.

33. Id., p. 36 : tableau ici remanié. 